\title{
The Utilization of Spironolactone in Heart Failure Patients at a Tertiary Hospital in Saudi Arabia
}

\author{
Abdulmalik S. Alotaibi ${ }^{1}$, Numan Alabdan ${ }^{1}$, Abdullah M. Alotaibi ${ }^{2}$, Haifa Aljaafary ${ }^{1}$, Mohammed \\ Alqahtani $^{2}$
}

1. Pharmaceutical Care, King Abdullah International Medical Research Center/King Saud bin Abdulaziz University for Health Sciences/King Abdulaziz Medical City - Ministry of National Guard Health Affairs, Riyadh, SAU 2. Medicine, King Abdullah International Medical Research Center/King Saud bin Abdulaziz University for Health Sciences/King Abdulaziz Medical City - Ministry of National Guard Health Affairs, Riyadh, SAU

Corresponding author: Abdulmalik S. Alotaibi, saifalotaibiab@gmail.com

\begin{abstract}
Introduction: Heart failure (HF) has high morbidity and mortality rates. Spironolactone has shown a 30\% reduction in all-cause mortality, reduction in hospitalizations, and sudden death. However, data shows low use of spironolactone in HF patients. We aim to assess spironolactone utilization in HF reduced Ejection Fraction (HFrEF) patients and to identify the factors affecting its prescribing.
\end{abstract}

Methods: A retrospective cross-sectional study of patients diagnosed with HF from January 2016 to January 2017 conducted at King Abdulaziz Medical City-Riyadh. Inclusion criteria: all adult $\mathrm{HFrEF}<40 \%$ who are eligible for spironolactone with New York Heart Association (NYHA) class II-IV. Serum creatinine should be $<2.5 \mathrm{mg} / \mathrm{dL}$ in men or $<2.0 \mathrm{mg} / \mathrm{dL}$ in women, or estimated glomerular filtration rate (eGFR) $>30$

$\mathrm{mL} / \mathrm{min} / 1.73 \mathrm{~m}^{2}$ and potassium $<5.0 \mathrm{mEq} / \mathrm{L}$. Exclusion criteria: pediatrics, end-stage renal disease, primary aldosteronism, and allergy to spironolactone.

Results: We screened around $5000 \mathrm{HF}$ patients, of whom 368 were included. Among 195 patients who were not on spironolactone, 121 patients were eligible to use it; however, they did not receive it. One hundred seventy-three patients were on spironolactone, of whom 30 received the drug although they did not meet the eligibility criteria. The mean age of patients on spironolactone was $61 \pm 14$ and the mean age of patients not on spironolactone was $66.6 \pm 15.6$. Two hundred seventy-seven patients in the study population were male. Regarding comorbidities, 265 patients were diabetic. As for laboratory findings, the mean potassium for patients on spironolactone was $4.3 \mathrm{mEq} / \mathrm{L}$; the creatinine and eGFR for patients on spironolactone were $82 \mathrm{umol} / \mathrm{L}(0.9 \mathrm{mg} / \mathrm{dl})$ and $88 \mathrm{~mL} / \mathrm{min} / 1.73 \mathrm{~m}^{2}$ while those not on spironolactone had higher creatinine at 93 umol/L (1 mg/dl) and eGFR $80 \mathrm{~mL} / \mathrm{min} / 1.73 \mathrm{~m}^{2}$. Using multivariate regression, we found many factors affecting spironolactone utilization, including EF before spironolactone, serum creatinine, angiotensinconverting enzyme inhibitors (ACEI), angiotensin-II receptor antagonists (ARBs), furosemide, statin, and stroke.

Received 08/07/2020

Review began 08/10/2020 Review ended 08/19/2020 Published 08/25/2020

๑) Copyright 2020

Alotaibi et al. This is an open access article distributed under the terms of the Creative Commons Attribution License CC-BY 4.0., which permits unrestricted use, distribution, and reproduction in any medium, provided the original author and source are credited.
Conclusions: Spironolactone for HFrEF is underutilized. EF before spironolactone, serum creatinine, ACEI, ARBs, furosemide, statin, and stroke significantly affect spironolactone utilization. Further studies are warranted to identify barriers affecting spironolactone utilization in HF patients from prescribers' perspectives.

Categories: Cardiology, Family/General Practice, Internal Medicine

Keywords: heart failure, spironolactone utilization, aldosterone antagonists, mineralocorticoid receptor antagonist, saudi arabia

\section{Introduction}

Heart failure (HF) is a common clinical condition with growing incidence and prevalence manifested by fatigue, dyspnea, and volume overload [1]. Worldwide, HF affects around 26 million people. In the United States (US) around 5.8 million people have HF. In addition, approximately $20 \%$ of the US population will develop HF in their lifetime [2]. Moreover, because of its increasing incidence, in 2030, HF will be diagnosed in one in every 33 US citizens, which could be linked to the aging population and the rate of survival for patients after myocardial infarction with current treatment options [3,4]. In Saudi Arabia, the estimated prevalence of HF is about 455,222 cases with an annual incidence of 32,200 cases [5]. Additionally, HF poses a tremendous burden with approximately 1.04 billion USD for a total of 320,000 HF-treated patients $(8,137$ USD annual cost per patient) [6]. Besides its high mortality and significant morbidity, HF patients will require multiple hospitalizations. It is responsible for nearly 1 million annual hospitalizations in the US and more than 12 million physician office visits every year [7,8]. Efforts have been done to optimally implement cost-effective and high-value therapies to improve overall provided care and reduce cost expenditures [9].

Currently, many therapeutic options exist for treating HF, including angiotensin-converting enzyme 
inhibitors (ACEIs), angiotensin-II receptor antagonists (ARBs), aldosterone antagonists (AA), ß-blockers (BB), and angiotensin receptor-neprilysin inhibitor (ARNI). Many trials have shown the added benefits of AA, commonly spironolactone, which has shown a $30 \%$ reduction in all-cause mortality, in addition to a reduction in hospitalizations and sudden death $[10,11]$. Based on that, it has a high-class recommendation by the American College of Cardiology/American Heart Association (ACCF/AHA) and the European Society of Cardiology (ESC) guidelines for chronic HF $[12,13]$. To promote the safe practice, guidelines have specified that before starting patients on spironolactone, serum creatinine levels should be $2.5 \mathrm{mg} / \mathrm{dL}$ or less and 2.0 $\mathrm{mg} / \mathrm{dL}$ or less in men and women, respectively. Also, serum potassium levels should be less than $5.0 \mathrm{mEq} / \mathrm{L}$. However, gaps in utilizing and monitoring spironolactone have been identified and addressed in many studies [14-18]. In a large 2009 observational study of more than 40,000 patients, less than one-third of eligible HF patients received AA [18]. Another study with a sample of 15,381 patients found that only $36 \%$ had a prescription of AA at discharge [19]. It has been suggested that the primary reasons for failure to prescribe AA are physicians' knowledge, familiarity, and agreement with guidelines [18]. In support of this hypothesis, another study found that $9 \%$ of primary care providers (PCPs) were not familiar with eplerenone [20]. Besides, the safety monitoring for hyperkalemia has been found inadequate as shown in the Randomized Aldactone Evaluation Study (RALES); since there was a noticeable increase in hospital admissions for hyperkalemia and in-hospital deaths [21]. One reason that may contribute to this finding is the lack of close monitoring of potassium levels in patients receiving spironolactone. Moreover, Shah et al. concluded that patients on spironolactone for $\mathrm{HF}$ do not receive needed follow-up of potassium or creatinine concentrations, taking into consideration that hyperkalemia and renal dysfunction are common [17].

These findings shed light on the aims of this study, which are to assess the utilization of spironolactone in indicated HF patients, to provide insights about the current practice in Saudi Arabia, and to identify factors limiting the use of AA.

\section{Materials And Methods}

This was a retrospective cross-sectional chart review of patients with HF diagnosis from January 2016 to January 2017 conducted at King Abdul-Aziz Medical City (KAMC) in Riyadh. Inclusion criteria involved: all adult HF patients with Ejection Fraction (EF) $<40 \%$ who are eligible for spironolactone (HF patients with New York Heart Association [NYHA] class II-IV. Should have creatinine $<2.5 \mathrm{mg} / \mathrm{dL}$ in men or $<2.0 \mathrm{mg} / \mathrm{dL}$ in women, or estimated glomerular filtration rate $[\mathrm{eGFR}]>30 \mathrm{~mL} / \mathrm{min} / 1.73 \mathrm{~m} 2$ and potassium $<5.0 \mathrm{mEq} / \mathrm{L}$ ). Exclusion criteria were: pediatrics, end-stage renal disease (since it is contraindicated to use spironolactone when creatinine clearance $<30 \mathrm{ml} / \mathrm{min}$ ), primary aldosteronism (since spironolactone can be used for different indication), and allergy to spironolactone. Outcomes variables include serum creatinine, potassium, eGFR, blood pressure (BP), whether the patient is on spironolactone, its dose, and if it is discontinued or not. The Institutional Review Board (IRB) approved our study (RC number: 17/101/R). After IRB approval, we queried data from a randomly selected sample of around $5000 \mathrm{HF}$ patients from January 2016 to January 2017 by using the electronic system BestCare to generate a list of patients to be screened to meet our inclusion and exclusion criteria. The final study population was 368 patients.

\section{Statistical analysis}

Descriptive statistics were used to describe the study population. Data were analyzed using the appropriate descriptive statistics for the primary and secondary endpoints. All analyses were done using Statistical Product and Service Solutions (SPSS) Statistics for Windows, version 23 (IBM Corp., Armonk, NY). Sociodemographic and clinical characteristics of participants are presented

according to spironolactone use (yes vs no). To compare continuous data (e.g. serum creatinine) for the two groups, an independent t-test was used. The Chi-Square test was used to evaluate nominal data (e.g. patient comorbidities). A p-value below 0.05 is considered statistically significant in this study.

\section{Multivariate regression}

analyses were performed to identify variables associated with spironolactone utilization. All variables were investigated including (age, gender, ejection fraction, NYHA classifications, body mass index, heart rate, systolic/diastolic blood pressure, history ( $\mathrm{Hx}$ ) of congestive HF or ischemic, diabetes mellitus, hypertension, dyslipidemia, smoking, asthma, atrial fibrillation, venous thromboembolism, stroke, chronic obstructive pulmonary disease, anemia, angina, unstable angina, myocardial infarction, family history ( $\mathrm{FHx}$ ) of coronary artery disease (CAD), medications: ACEI, ARBs, BB, aspirin, clopidogrel, furosemide, hydrochlorothiazide, indapamide, statin, hydralazine, isosorbide dinitrate, warfarin, and digoxin; and labs: sodium, potassium, creatinine, eGFR, and B-type natriuretic peptide (BNP). After that, due to the small sample size, we did backward elimination to omit factors that were not significantly associated with spironolactone use to end up with the following factors (age, EF, systolic blood pressure [SBP], diabetes, anemia, FHx of CAD, ACEI, furosemide, indapamide, statin, hydralazine, warfarin, digoxin, potassium, creatinine, and eGFR).

\section{Results}

Between January 2016 and January 2017, 368 HFrEF patients were included from the screening of around 5000 heart failure patients. The use of spironolactone was 173 (47.01\%) patients while 195 (52.99\%) were not on it. 


\section{Cureus}

Baseline characteristics of the study population are shown in Table 1 . The mean age of patients on spironolactone was $61 \pm 14$ and the mean age of patients not on spironolactone was $66.6 \pm 15.6$. Two hundred and seventy-seven patients in the study population were male. As for clinical variables, those who had EF $<30$ had more likelihood of going on spironolactone than patients with EF 30-40 where more were not on it. Also, patients who were on spironolactone had more control on SBP 121 1 19. In comorbidities, 265 patients in the study population were diabetic. Additionally, patients with an FHx of CAD had more prescriptions for spironolactone (12 patients) than those with a negative FHx (three patients). Moreover, medications that were used more concomitantly with spironolactone were ACEI, furosemide, warfarin, and digoxin while indapamide, statins, and hydralazine were used less concomitantly. As for laboratory findings, the mean serum potassium for patients on spironolactone was $4.3 \mathrm{mEq} / \mathrm{L}$; the creatinine and eGFR for patients on spironolactone $82 \mathrm{umol} / \mathrm{L}(0.9 \mathrm{mg} / \mathrm{dl})$ and $88 \mathrm{~mL} / \mathrm{min} / 1.73 \mathrm{~m} 2 \mathrm{eGFR}$ while those not on spironolactone had higher creatinine $93 \mathrm{umol} / \mathrm{L}(1 \mathrm{mg} / \mathrm{dl})$ and $80 \mathrm{~mL} / \mathrm{min} / 1.73 \mathrm{~m} 2$ eGFR; the BNP was higher in patients on spironolactone $153 \mathrm{pmol} / \mathrm{L}$ and $109 \mathrm{pmol} / \mathrm{L}$ in patients not on spironolactone.

Table 1

Baseline Characteristics

Variables

On Spironolactone $\mathrm{n}=173$

Not On Spironolactone $\mathrm{n}=195$

Demographics

$61.0(14.2)$

$66.6(15.7)$

P-Value

Age ( \pm SD), years

$61.0(14.2)$

$<0.01$

Gender

Male n (\%)

125 (33.9)

$152(41.3)$

0.20

Clinical Variables

Ejection Fraction Before Spironolactone

$<30$ n (\%)

$85(23.1)$

58 (15.8)

$<0.01$

$30-40$ n (\%)

88 (23.9)

137 (37.2)

SBP ( $\pm \mathrm{SD}), \mathrm{mmHg}$

120.9 (19.2)

125.7 (18.1)

0.01

Co-Morbidities

Diabetes

Yes n (\%)

$113(30.7)$

152 (41.3)

Anemia

Yes n (\%)

4 (1.09)

Family History of CAD

Yes n (\%)

$12(3.26)$

Medications

ACEI

Yes n (\%)

112 (30.4)

105 (28.5)

Furosemide

Yes n (\%)

130 (35.3)

96 (26.1)

$<0.01$

Indapamide

Yes n (\%)

$1(0.27)$

7 (1.90)

Statin

Yes n (\%)

151 (41.0)

$184(50.0)$

0.01

Hydralazine

Yes n (\%)

5 (1.36)

19 (5.16)

$<0.01$

Warfarin 


\section{Cureus}

$\begin{array}{lll}\text { Yes n }(\%) & 22(5.98) & 11(2.99)\end{array}$

Digoxin

Yes n (\%)

$13(3.53)$

$6(1.63)$

0.06

Laboratory Findings

Baseline Potassium - mEq/L

$4.3(0.41)$

$4.4(0.42)$

0.07

Baseline Creatinine - umol/L

$81.2(23.4)$

$92.7(38.3)$

$<0.01$

Baseline eGFR - mL/min/1.73m²

$88.0(23.7)$

$79.6(27.3)$

$<0.01$

Baseline BNP pmol/L

$153(218)$

109 (173)

0.06

\section{TABLE 1: Baseline Characteristics}

SBP: systolic blood pressure, SD: standard deviation, CAD: coronary artery disease, ACEI: angiotensin-converting enzyme inhibitor, eGFR: estimated glomerular filtration rate, BNP: B-type natriuretic peptide

As for the utilization of spironolactone (Figure 1), among 195 patients who were not on spironolactone, 121 patients had eligibility to use it; however, they did not receive it. One hundred seventy-three patients were on spironolactone, of whom 30 patients received the drug although they did not meet the eligibility criteria.

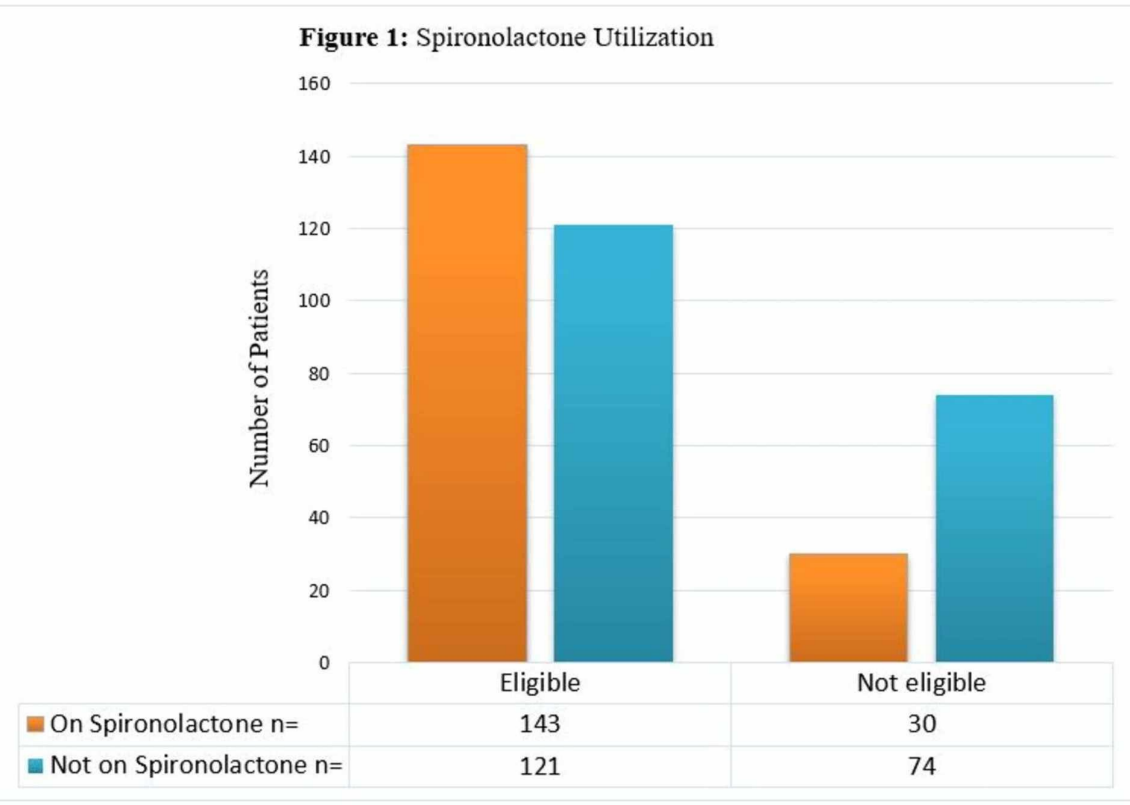

\section{FIGURE 1: Spironolactone Utilization}

Moreover, in Table 2, using multivariate logistic regression to find independent factors associated with the underutilization of spironolactone, we found the following factors to be significant: $\mathrm{EF}$ before spironolactone (odds ratio [OR] $=2.56$ [95\% CI: $1.40-4.69, \mathrm{P}=<0.01]$ ), serum creatinine $(\mathrm{OR}=1.01[95 \% \mathrm{CI}$ : 1.00-1.02, $\mathrm{P}=0.02]$ ), ACEI (OR=7.46 [95\% CI: 2.45-22.7, $\mathrm{P}=<0.01]$ ), ARBs (OR=6.54 [95\% CI: 2.01-21.3, $\mathrm{P}=$ $<0.01]$ ), furosemide ( $\mathrm{OR}=3.54$ [95\% CI: 1.90-6.62, $\mathrm{P}=<0.01]$ ), statins ( $\mathrm{OR}=0.18$ [95\% CI: 0.05-0.61, $\mathrm{P}=<0.01]$ ), stroke (OR=2.86 [95\% CI: $1.00-8.17, \mathrm{P}=0.05])$. 


\section{Cureus}

\begin{tabular}{|c|c|c|}
\hline Variables & Odds Ratio (95\% Cl) & P-Value \\
\hline Ejection Fraction Before Spironolactone & $2.56(1.40-4.69)$ & $<0.01$ \\
\hline Stroke & $2.86(1.00-8.17)$ & 0.05 \\
\hline ACEI & $7.46(2.45-22.7)$ & $<0.01$ \\
\hline ARBS & $6.54(2.01-21.3)$ & $<0.01$ \\
\hline Furosemide & $3.54(1.90-6.62)$ & $<0.01$ \\
\hline Statin & $0.18(0.05-0.61)$ & $<0.01$ \\
\hline Serum Creatinine & $1.01(1.00-1.02)$ & 0.02 \\
\hline
\end{tabular}

\section{TABLE 2: Independent Association Using Logistic Regression}

ACEI: angiotensin-converting enzyme inhibitor, ARBS: angiotensin-II receptor antagonists

\section{Discussion}

To the best of our knowledge, this is the first study to assess spironolactone utilization in HFrEF patients in Saudi Arabia. In this cross-sectional retrospective study, spironolactone was underutilized for indicated HF patients. The factors associated with the underutilization of spironolactone were EF, serum creatinine (although it was within an acceptable range to use spironolactone), ACEI, ARBs, furosemide, statins, and stroke.

Spironolactone underutilization in this study is consistent with previous studies. In a registry that was done between 2005-2007, they found that patients eligible to use AA represent only $32.5 \%$ of the study population [18]. In the Improve the Use of Evidence-Based Heart Failure Therapies in the Outpatient Setting (IMPROVE HF) registry, the use of AA was to 36\% of 2505 eligible patients [19]. Similarly, in the EuroHeart Failure Survey II (EHFS II), 47.5\% of patients used AA upon discharge after admission with HF [22]. Furthermore, in findings from BIOSTAT-CHF, a European multicenter, prospective observational study, only $56 \%$ of eligible patients received AA at baseline which improved to $63 \%$ after following up with patients as part of an HF treatment optimization program [23]. Also, in the Medicare-linked Organized Program To Initiate Lifesaving Treatment In Hospitalized Patients With Heart Failure (OPTIMIZE-HF) registry, 6986 from 8206 patients had eligibility to use spironolactone based on serum creatinine; however, they were not on it [24]. Additionally, in the Swedish Heart Failure Registry (SwedeHF) 11,215 patients, only 4443 (40\%) of eligible patients received an $\mathrm{AA}$, and to find factors associated with underutilization of AA where creatinine was found to be significantly associated with low-use (although within an acceptable range) while serum potassium was not significant [25]. In our study, the rate of using spironolactone in eligible HF patients was 39\%.

Several factors have been associated with spironolactone underutilization in this study, including EF before spironolactone, since patients with HFrEF have a stronger recommendation to use AA to improve survival; another factor is serum creatinine, as the hyperkalemia risk increases with worsening renal function; ACEI and ARBs both limited the use of spironolactone due to the effect of hyperkalemia they can cause; furosemide, which could be due to the risk of over diuresis/dehydration, statin, and stroke; where most patients are on ACEI/ARBs leading to an increased risk of hyperkalemia [26]. In addition to the factors limiting spironolactone use, we plan to do another study to find barriers limiting spironolactone utilization from the prescribers' perspective, assessing the knowledge, perception, and comprehension of the ACCF/AHA and ESC guidelines in regard to AA use among HF patients.

This study has some limitations that need to be identified, for example the small sample size of the study population, in addition to the single-center setting, although the institution (KAMC) is considered one of the leading hospitals in Saudi Arabia in cardiovascular medicine. Another limitation is the retrospective nature of the study, therefore the causality effect cannot be applied. Moreover, when using regression, a stepwise selection was utilized due to the lack of sufficient sample size, which might have an effect on the accuracy of the results.

\section{Conclusions}

The use of spironolactone for HF patients is underutilized. EF before spironolactone, serum creatinine, ACEI, ARBs, furosemide, statin, and stroke significantly affected spironolactone utilization. The findings of this study indicate that there is a gap in utilizing spironolactone in Saudi Arabia, which implicates the necessity to find barriers limiting its use and make protocols to assure the compliance with the guidelines. Further studies are warranted to identify other factors and potential barriers from the prescribers' perspectives 


\section{Additional Information Disclosures}

Human subjects: Consent was obtained by all participants in this study. King Abdullah International Medical Research Center issued approval RC number: 17/101/R. The study was approved by the Institutional Review Board (IRB) from King Abdullah International Medical Research Cente. The approval RC number: 17/101/R. Animal subjects: All authors have confirmed that this study did not involve animal subjects or tissue. Conflicts of interest: In compliance with the ICMJE uniform disclosure form, all authors declare the following: Payment/services info: All authors have declared that no financial support was received from any organization for the submitted work. Financial relationships: All authors have declared that they have no financial relationships at present or within the previous three years with any organizations that might have an interest in the submitted work. Other relationships: All authors have declared that there are no other relationships or activities that could appear to have influenced the submitted work.

\section{References}

1. Ahmed A: American College of Cardiology/American Heart Association Chronic Heart Failure Evaluation and Management guidelines: relevance to the geriatric practice. J Am Geriatr Soc. 2003, 51:123-6. 10.1034/j.1601-5215.2002.51020.x

2. Lloyd-Jones DM: The risk of congestive heart failure: sobering lessons from the Framingham Heart Study . Curr Cardiol Rep. 2001, 3:184-90. 10.1007/s11886-001-0021-1

3. Heidenreich PA, Albert NM, Allen LA, et al.: Forecasting the impact of heart failure in the United States: a policy statement from the American Heart Association. Circ Heart Fail. 2013, 6:606-19. 10.1161/HHF.0b013e318291329a

4. Lopez-Sendon J, Montoro N: The changing landscape of heart failure outcomes . Medicographia. 2015, 37:125-34.

5. AbuRuz ME, Alaloul F, Saifan A, Masa'deh R, Abusalem S: Quality of life for Saudi patients with heart failure: a cross-sectional correlational study. Glob J Health Sci. 2015, 25:49-58. 10.5539/gjhs.v8n3p49

6. AlHabeeb, W. Akhras, K. AlGhalayini, et al.: Understanding heart failure burden in Middle East countries: economic impact in Egypt, Saudi Arabia and United Arab Emirates. Value Heal. 2018, 21:123. 10.1016/j.jval.2018.04.840

7. Hall MJ, Levant S, DeFrances CJ: Hospitalization for congestive heart failure: United States, 2000-2010. NCHS Data Brief. 2012, 108:1-8.

8. Allen LA, Tang F, Jones P, Breeding T, Ponirakis A, Turner SJ: Signs, symptoms, and treatment patterns across serial ambulatory cardiology visits in patients with heart failure: insights from the NCDR PINNACLE ${ }^{\circledR}$ registry. BMC Cardiovasc Disord. 2018, 18:80. 10.1186/s12872-018-0808-2

9. Berry C, Murdoch DR, McMurray JJ: Economics of chronic heart failure . Eur J Heart Fail. 2001, 3:283-91. 10.1016/S1388-9842(01)00123-4

10. Pitt B, Zannad F, Remme WJ, et al.: The effect of spironolactone on morbidity and mortality in patients with severe heart failure. N Engl J Med. 1999, 341:709-17. 10.1056/NEJM199909023411001

11. Ezekowitz JA, McAlister FA: Aldosterone blockade and left ventricular dysfunction: a systematic review of randomized clinical trials. Eur Heart J. 2009, 30:469-77. 10.1093/eurheartj/ehn543

12. Yancy CW, Jessup M, Bozkurt B, et al.: 2017 ACC/AHA/HFSA focused update of the 2013 ACCF/AHA Guideline for the Management of Heart Failure: a report of the American College of Cardiology/American Heart Association Task Force on Clinical Practice Guidelines and the Heart Failure Society of America. Circulation. 2017, 136:137-61. 10.1161/CIR.0000000000000509

13. Ponikowski P, Voors AA, Anker SD, et al.: 2016 ESC Guidelines for the diagnosis and treatment of acute and chronic heart failure: The Task Force for the diagnosis and treatment of acute and chronic heart failure of the European Society of Cardiology (ESC). Eur Heart J. 2016, 37:2129-200. 10.1093/eurheartj/ehw128

14. Masoudi FA, Gross CP, Wang Y, et al.: Adoption of spironolactone therapy for older patients with heart failure and left ventricular systolic dysfunction in the United States, 1998-2001. Circulation. 2005, 112:3947. 10.1161/CIRCULATIONAHA.104.527549

15. Bozkurt B, Agoston I, Knowlton A: Complications of inappropriate use of spiroholactone in heart failure: when an old medicine spirals out of new guidelines. J Am Coll Cardiol. 2003, 15:211-4. 10.1016/S07351097(02)02694-3

16. Dev S, Lacy ME, Masoudi FA, Wu W-C: Temporal trends and hospital variation in mineralocorticoid receptor antagonist use in veterans discharged with heart failure. J Am Heart Assoc. 2015, 4:10.1161/JAHA.115.002268

17. Shah KB, Rao K, Sawyer R, et al.: The adequacy of laboratory monitoring in patients treated with spironolactone for congestive heart failure. J Am Coll Cardiol. 2005, 46:845-9. 10.1016/j.jacc.2005.06.010

18. Albert NM, Yancy CW, Liang L, et al.: Use of aldosterone antagonists in heart failure . JAMA. 2009, 302:1658-65. 10.1001/jama.2009.1493

19. Fonarow GC, Yancy CW, Albert NM, et al.: Heart failure care in the outpatient cardiology practice setting: findings from IMPROVE HF. Circ Heart Fail. 2008, 1:98-106. 10.1161/CIRCHEARTFAILURE.108.772228

20. Dev S, Hoffman TK, Kavalieratos D, et al.: Barriers to adoption of mineralocorticoid receptor antagonists in patients with heart failure: a mixed-methods study. J Am Heart Assoc. 2016, 5:002493. 10.1161/JAHA.115.002493

21. Juurlink DN, Mamdani MM, Lee DS, et al.: Rates of hyperkalemia after publication of the randomized aldactone evaluation study. N Engl J Med. 2004, 5:543-51. 10.1056/NEJMoa040135

22. Nieminen MS, Brutsaert D, Dickstein K, et al.: EuroHeart Failure Survey II (EHFSII): a survey on hospitalized 


\section{Cureus}

acute heart failure patients: description of population. Eur Heart J. 2006, 27:2725-36.

10.1093/eurheartj/ehl193

23. Ferreira JP, Rossignol P, Machu J-L, et al.: Mineralocorticoid receptor antagonist pattern of use in heart failure with reduced ejection fraction: findings from BIOSTAT-CHF. Eur J Heart Fail. 2017, 19:1284-93. 10.1002/ejhf.900

24. Bayoumi E, Lam PH, Dooley DJ, et al.: Spironolactone and outcomes in older patients with heart failure and reduced ejection fraction. Am J Med. 2018, 132:71-80. 10.1016/j.amjmed.2018.09.011

25. Savarese G, Carrero J-J, Pitt B, et al.: Factors associated with underuse of mineralocorticoid receptor antagonists in heart failure with reduced ejection fraction: an analysis of 11215 patients from the Swedish Heart Failure Registry. Eur J Heart Fail. 2018, 20:1326-34. 10.1002/ejhf.1182

26. Abbas S, Ihle P, Harder S, et al.: Risk of hyperkalemia and combined use of spironolactone and long-term ACE inhibitor/angiotensin receptor blocker therapy in heart failure using real-life data: a population- and insurance-based cohort. Pharmacoepidemiol Drug Saf. 2015, 1:24. 10.1002/pds.3748 